\title{
Anatomic Relationships Between the Coronary Venous System, Surrounding Structures, and the Site of Origin of Epicardial Ventricular Arrhythmias
}

\author{
THOMAS P. CARRIGAN, M.D., F.H.R.S., SMITA PATEL, M.D., MIKI YOKOKAWA, M.D., \\ ERIC SCHMIDLIN, M.D., SCOTT SWANSON, PH.D., FRED MORADY, M.D., and \\ FRANK BOGUN, M.D.
}

From the Division of Cardiovascular Medicine and Department of Radiology, University of Michigan, Ann Arbor, Michigan, USA

\begin{abstract}
Epicardial VT. Background: Epicardial left ventricular (LV) idiopathic ventricular arrhythmias (VAs) can be approached via the pericardial space, the coronary venous system (CVS), or other surrounding structures. The anatomic relationships between epicardial sites of origin (SOO) of VAs and surrounding anatomic structures have not been systematically described.

Methods and Results: In 17 patients with idiopathic epicardial VAs, the relationships between the SOO and the CVS and other neighboring anatomic structures were assessed by computed tomographic angiography. Ablation was successful in $12 / 17$ patients (71\%). In 10/17 patients, the SOO was at a distance of $\leq 4 \mathrm{~mm}$ from a coronary artery. The SOO was closer to the CVS $(2.1 \pm 1.5 \mathrm{~mm})$ than to the pericardial space $(9.7$ $\pm 3.7 \mathrm{~mm})$ or the $L V$ endocardium $(7.7 \pm 2.7 \mathrm{~mm})$. Successful ablations were carried out from the CVS $(n=3)$, the CVS and LV endocardium $(n=5)$, the CVS and the aortic cusp $(n=1)$, the CVS, the LV endocardium, and the aortic cusp $(n=1)$, the LV endocardium $(n=1)$, and the CVS and the pericardial space $(n=1)$. In the remaining 5 patients, a subxyphoid pericardial ablation procedure was attempted and failed in all 5 patients.

Conclusion: The CVS is closer to the SOO of epicardial idiopathic VAs than the pericardial space, the ventricular endocardium, and the aortic cusps. Given the proximity to coronary arteries at the SOO, radiofrequency energy often cannot be safely delivered to eliminate a VA and ablation may also need to be performed from adjacent structures. A subxyphoid pericardial ablation procedure has a low probability of success in patients with idiopathic epicardial VAs. (J Cardiovasc Electrophysiol, Vol. 25, pp. 1336-1342, December 2014)
\end{abstract}

catheter ablation, coronary venous system, epicardial ablation, idiopathic ventricular tachycardia, ventricular tachycardia

\section{Background}

Idiopathic ventricular arrhythmias (VAs) can arise from the left ventricular epicardium. Different approaches have been described for radiofrequency ablation including a subxyphoid pericardial approach, ${ }^{1}$ an approach via the coronary venous system (CVS), ${ }^{2}$ and a combined approach via adjacent anatomical structures including the CVS and the ventricular endocardium or the aortic cusps. ${ }^{3}$ We hypothesized that the CVS is closer to the epicardial myocardium where VAs originate from than is the pericardial space. The proximity of actual and potential ablation sites within the CVS to the left ventricular epicardium has not been described systematically. The purpose of this report was to describe the anatomic relationships at the site of origin (SOO) of epicardial VAs to the CVS, the pericardial space, the coronary arteries, and other surrounding structures.

Dr. Bogun received a grant from the Leducq Foundation.

No disclosures.

Address for correspondence: Frank Bogun, M.D., Cardiovascular Center, SPC 58531500 E. Medical Center Drive, Ann Arbor, MI 48109, USA. Fax: 734-936-7026; E-mail: fbogun@med.umich.edu

Manuscript received 30 April 2014; Revised manuscript received 18 June 2014; Accepted for publication 8 July 2014.

doi: $10.1111 /$ jce. 12497
Methods

\section{Patient Characteristics (Table 1)}

The study subjects consisted of 17 consecutive patients (12 males, age $51 \pm 14$ years, ejection fraction: $46 \pm 12 \%$ ) referred for ablation of VAs originating from the epicardium. Patients were included in this study if they had idiopathic VAs with an epicardial SOO based on intracardiac mapping. Two patients had prior myocardial infarctions. The patients had cardiac magnetic resonance imaging prior to the procedure. In the 2 patients with prior myocardial infarction, the location of the scar was not epicardial and did not involve the SOO of the VAs. The VAs consisted of PVCs in 8 patients and VT in 9 patients. Twelve of 17 patients $(71 \%)$ had previously failed ablation procedures. Patients had a mean of $2.4 \pm 3$ different VAs (range 1-11). The predominant VA was epicardial in origin.

The patients had not responded to a mean of $1.4 \pm 1$ antiarrhythmic medications. Cardiac-computed tomographic angiography was performed to evaluate the coronary arteries and the CVS as well as the anatomic relation to adjacent structures.

\section{SOO and 12-Lead ECG}

Twelve-lead electrocardiograms (ECG) of the VAs were recorded in all patients. The ECG was analyzed for bundle 

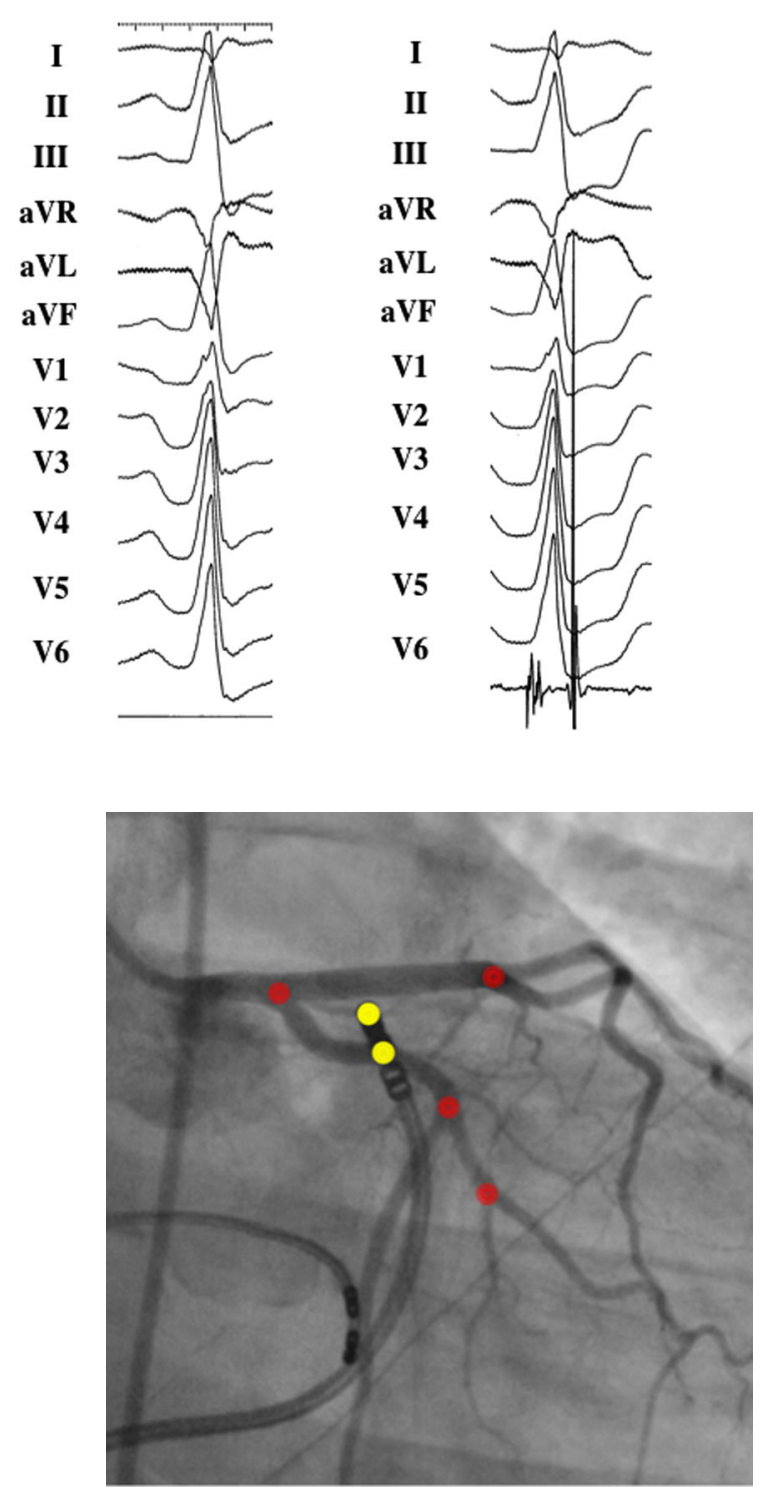

Figure 2. Right anterior oblique view of the left main coronary artery with its bifurcation into the left anterior descending and circumflex artery. The ablation catheter and distal recording electrodes are marked with yellow tags. The ablation catheter is at the site of origin of the ventricular arrhythmia. Bifurcation sites of the coronary artery are marked with red tags indicating fiducial points that were used for image registration to the CTA images. For a high quality, full color version of this figure, please see Journal of Cardiovascular Electrophysiology's website: www. wileyonlinelibrary. com/journal/jce

branch block morphology, axis, QRS width, and the precordial transition (before or after V1). $\mathrm{R}$ wave and $\mathrm{S}$ wave width as well as percentage of the $R$ wave relative to the QRS duration were measured in leads V1 and V2. The SOO was defined as the earliest activation time obtained during mapping in the presence of a matching pace-map ( $\geq 10 / 12$ leads) at this site (Fig. 1). An epicardial SOO was defined as an early activation time combined with a matching pace-map from within the CVS or the epicardial space. The epicardial myocardium closest to the location of the mapping catheter where these criteria were met was defined as the SOO. An epicardial SOO was identified in all patients.

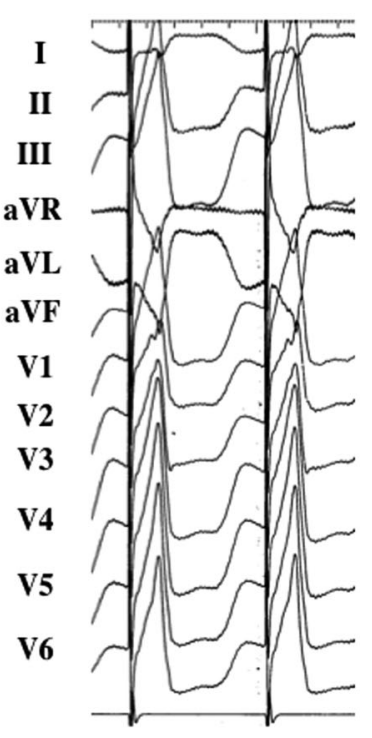

\begin{abstract}
Figure 1. Left panel: Twelve-lead ECG of a spontaneous VT with a right bundle branch block morphology. Middle panel: Local activation time (-27 milliseconds) at the site of origin within the great cardiac vein (GCV). Right panel: Pacing in the distal GCV results in a QRS morphology that matches with the QRS morphology of the induced VT.
\end{abstract}

\section{Electrophysiology Study and Mapping}

After informed consent was obtained, several multipolar catheters were introduced into the femoral veins and positioned in the His bundle position and the right ventricular apex. Arterial access was obtained in the femoral artery. After vascular access was obtained, 3,000 units of heparin was given and an ACT of $>250$ seconds was targeted with additional heparin administration. Programmed stimulation was repeated during infusion of isoproterenol. Electrograms were filtered from 50 to $500 \mathrm{~Hz}$. Recordings of the intracardiac electrograms were archived on disc (EP Med Systems, West Berlin, NJ, USA). An electroanatomical mapping system (CARTO, Biosense Webster, Diamond Bar, CA, USA) was used to guide mapping. Activation mapping was performed during VAs: PVCs or VT. Mapping was performed in the following sequence: For right bundle branch block VAs, the first anatomic structure that was mapped was the CVS followed by the aortic cusp, the left ventricular endocardium, and the right ventricular outflow tract. In patients with a left bundle branch block VA morphology, mapping was first performed in the right ventricular outflow tract, followed by the CVS, the aortic cusp, and the left ventricular endocardium. Pacing was performed (10-20 mA with a pulse width of 2 milliseconds) at the earliest site of activation, and if there was a matching pace-map, this was defined as the SOO. The coronary arteries were injected while the ablation catheter was at the SOO. Pacing was performed at high output $(20 \mathrm{~mA}$ at 2 milliseconds pulse width) to assess for phrenic nerve capture. If the distance to the closest coronary artery was $>4 \mathrm{~mm},{ }^{4}$ radiofrequency energy was applied at a power of 20-30 W using an open irrigated-tip catheter (Thermocool, Biosense Webster) targeting an impedance drop of $10 \mathrm{Ohms}$ for 60 seconds. If this eliminated the VA, an additional RF application of 60 seconds was delivered. If ablation failed to eliminate the VA, the aortic cusp and the mitral annulus were mapped. At the site closest to the SOO, pace-mapping was performed and radiofrequency energy was delivered. If this failed to eliminate the VA, a subxyphoid pericardial mapping procedure was attempted. Coronary angiography was repeated at the conclusion of the procedure. The successful site was defined as the target site that eliminated the VA. 


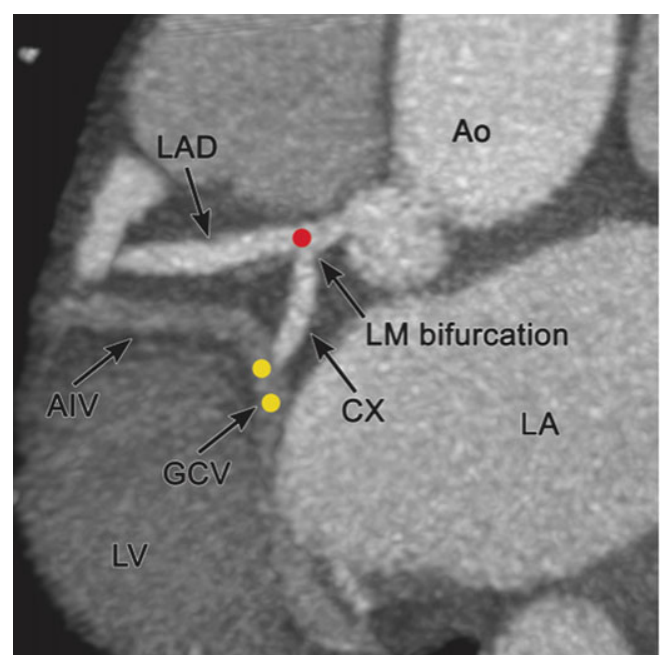

Figure 3. Multiplanar oblique reformatted view (2D) of the CTA at the level of the bifurcation of the left main coronary artery with the registered fiducial point from the coronary angiography (Fig. 2). Arrows indicate the great cardiac vein $(G C V)$, the anterior interventricular vein $(A I V)$, the left main $(L M)$ coronary artery with its bifurcation (Bif). The left ventricle ( $L V)$, the left atrium (LA), and the aorta (Ao) are also indicated. Location of the ablation catheter and distal recording electrodes are marked with yellow tags. For a high quality, full color version of this figure, please see Journal of Cardiovascular Electrophysiology's website: www. wileyonlinelibrary. com/journal/jce

If this occurred in the CVS, the CVS was defined as the successful site. If this occurred in the left ventricular endocardium after ablation within the CVS had failed, the successful site was defined as the CVS and the left ventricular endocardium.

\section{Cardiac-Computed Tomographic Angiography}

All patients underwent an ECG-gated contrast-enhanced CT angiography (CTA) scan using a 64 slice CT scanner (Discovery GE 750 HD, GE Healthcare, Milwaukee, WI, USA). Following a timing bolus with $15 \mathrm{~mL}$ of iopamidol (Isovue, Bracco Imaging, Singen, Germany) nonionic intra- venous contrast medium at $5 \mathrm{~mL} / \mathrm{second}$, a scan delay of peak plus 6 seconds was used to obtain the dedicated coronary CTA. Acquisition was performed at end diastole following an intravenously administered bolus via an antecubital vein of $80 \mathrm{~mL}$ iopamidol nonionic intravenous contrast medium followed by $50 \mathrm{~mL}$ saline at $5 \mathrm{~mL} / \mathrm{second}$ using a triphasic bolus power injector (MedRad Stellant MedRad Inc., Warrendale, PA, USA). In a post hoc analysis, the relationship between the CVS and neighboring anatomic structures was assessed. When the catheter was at the SOO, the left coronary artery was injected and the distance to the closest coronary artery was determined using biplane angiograms (Fig. 2). The tip and the distal electrodes of the ablation catheter were also identified on the 2D angiograms (Fig. 2). The angiogram with the catheter at the SOO was registered into the coronary CTA by using arterial bifurcations as fiducial points in enddiastole (Figs. 2 and 3). Using the locations of these fiducial markers and the angle between the cameras, 3D coordinates of the branch points and the catheter were calculated. The same branching points were located on a 3D CT angiogram study (Fig. 3). Using customized algorithms (Matlab, The Mathworks, Natick, MA, USA), the branching points from the $2 \mathrm{D}$ angiograms were translated and rotated by a leastsquare minimization routine to align with the branch points of the 3D CTA study. The position of the catheter was then translated and rotated by the same procedure to locate the catheter on the 3D CTA study. The positional error was 3.5 $\pm 2.2 \mathrm{~mm}$. The position of the catheter was visualized by superposition of the catheter points onto the 3D CT angiogram data (Fig. S1). The location of the catheter tip at the SOO of the VA was also registered to the CT angiogram and multiple measurements were performed on the CTA studies on an Advantage Windows workstation, version 4.5 (GE Healthcare) from this location (Figs. 4 and 5):

(1) Distance of the CVS to the closest coronary artery (Fig. 4a) when the ablation catheter is at the SOO.

(2) Distance of the SOO to the closest site of the visceral pericardial surface (Fig. 4b).

(3) Distance of the SOO to the left ventricular endocardium (Fig. 4c).
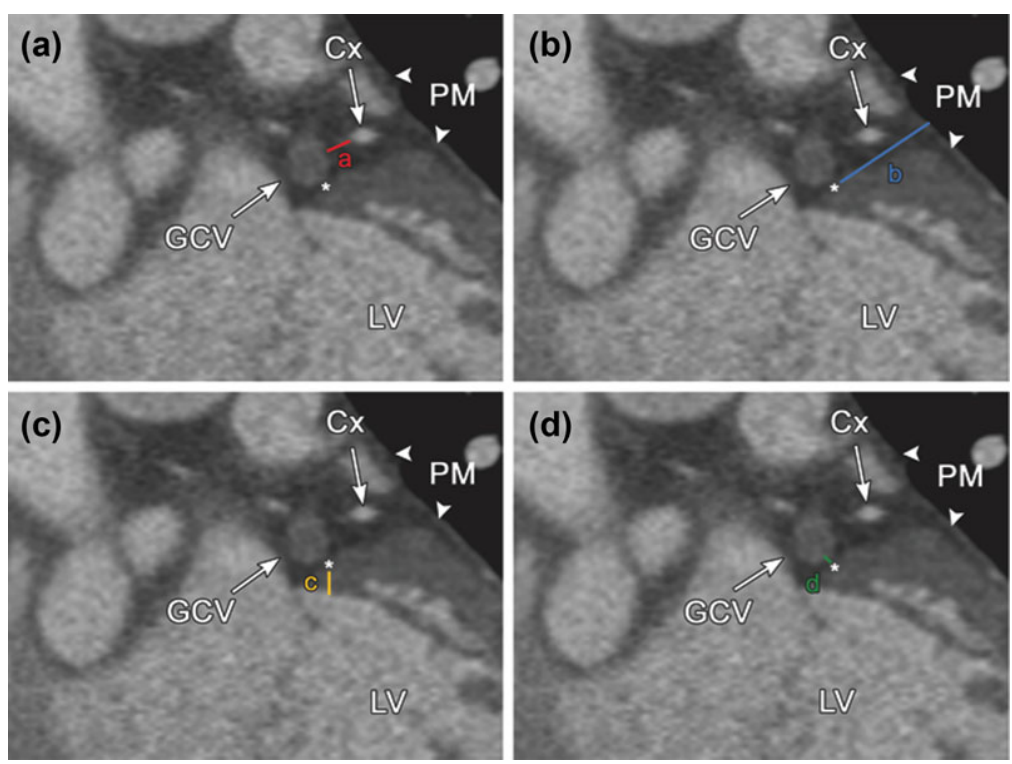

Figure 4. $(a-d)$ Show the distance of the adjacent anatomic structures at the site of origin: the great cardiac vein $(G C V)$, the left ventricle $(L V)$, the circumflex coronary artery $(C x)$, and the pericardial membrane (PM) are indicated. The distance from the GCV to the closest coronary artery (a) was measured when the catheter was at the site of origin (white asterisk). The distance is $1.0 \mathrm{~mm}$ to the circumflex artery (11 o'clock). The distance from the site of origin of the ventricular arrhythmia to the pericardial membrane (b) was $13.7 \mathrm{~mm}$ and the distance to the closest endocardial site (c) was 5.2 $\mathrm{mm}$. Finally, the distance of the GCV to the sit of origin was $1.4 \mathrm{~mm}(d)$. The ablation procedure in this patient was successful from the GCV. For a high quality, full color version of this figure, please see Journal of Cardiovascular Electrophysiology's website: www.wileyonlinelibrary. com/journal/jce 


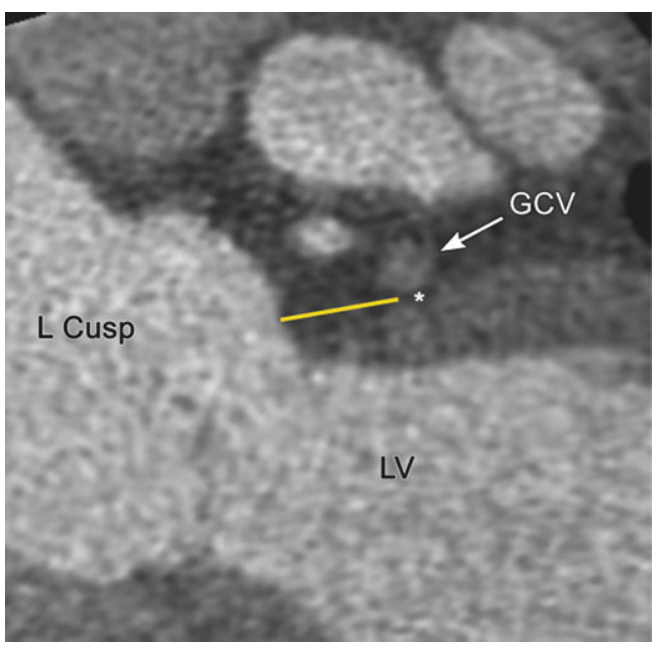

Figure 5. Multiplanar oblique reformat of the CTA shows the great cardiac vein $(G C V)$, the left ventricle ( $L V)$, and the left aortic cusps ( $L C u s p)$. The distance from the site of origin (asterisk) to the aortic cusp was measured (yellow line) and was $12.4 \mathrm{~mm}$. For a high quality, full color version of this figure, please see Journal of Cardiovascular Electrophysiology's website: www.wileyonlinelibrary.com/journal/jce

(4) Distance of the CVS to the SOO (Fig. 4d).

(5) Distance of the SOO to the aortic cusp (Fig. 5).

Finally, the distance of the CVS along the great cardiac vein (GCV) - including the distal part of the GCV and the beginning of the anterior interventricular vein (AIV) - to surrounding anatomic structures (coronary artery, visceral pericardium, left ventricular epicardium, left ventricular endocardium, and aortic cusp) was measured (Fig. S1, Table S1) in $5 \mathrm{~mm}$ increments. The measurements were started from the left main coronary bifurcation by $5 \mathrm{~mm}$ decrements up to $3 \mathrm{~cm}$ in the direction of the coronary sinus and also in 5 $\mathrm{mm}$ increments from the left main bifurcation up to $3 \mathrm{~cm}$ into the direction of the AIV. The same measurements were made for the middle cardiac vein starting from its origin at the coronary sinus (Table S2).

The analysis was performed by 2 investigators (TC and ES) who were blinded to the procedure details. Interobserver agreement for the measured distances with an accuracy of $\leq 0.5 \mathrm{~mm}$ showed a kappa value of 0.88 . Using commercially available software packages (AW Volumeshare 4, General Electric), images were analyzed in both 2D and 3D planes. The GCV was processed from the os of the coronary sinus to the distal aspect of the (Fig. S1).

\section{Statistical Analysis}

Categorical variables were compared using the chi-square or Fisher's exact test, as appropriate. If a cell size was $<5$, the Fisher's exact test was used. Normally distributed continuous variables were reported as mean and standard deviation. Multiple comparisons of categorical data were reported with the Bonferroni correction. A P-value $<0.05$ was considered significant.

\section{Follow-Up}

Patients were followed at an outpatient clinic 3 months after ablation and thereafter every $12-48$ months. Holter monitor was performed at 3 months postablation to assess the
TABLE 1

Patient Characteristics

\begin{tabular}{lc}
\hline Age (years) & $\mathbf{5 1} \pm \mathbf{1 4}$ \\
\hline Gender (m/f) & $12 / 5$ \\
Arrhythmia (n; PVC/VT) & $8 / 9$ \\
Ejection fraction (\%) & $46 \pm 12$ \\
Failed antiarrhythmic medications (n) & $1.4 \pm 1$ \\
Prior failed ablation procedure & $12 / 17$ \\
PVC burden (\%) & $28 \pm 9$ \\
VA morphology & \\
LBBBIA & 7 \\
LBBBSA & 2 \\
RBBBIA & 8 \\
RBBBSA & 0 \\
\hline
\end{tabular}

PVC = premature ventricular complex; VT = ventricular tachycardia; VA $=$ ventricular arrhythmia; LBBIA = left bundle branch block inferior axis morphology; LBBSA = left bundle branch block superior axis morphology; RBBIA = right bundle branch block inferior axis morphology; RBBSA = right bundle branch block superior axis morphology.

PVC burden. Antiarrhythmic medications were discontinued in all patients with effective ablation procedures.

\section{Results}

\section{Acute Ablation Outcome and Mapping Data}

The ablation procedure was successful in $12 / 17$ patients (71\%). The mean activation time at the SOO was $-30 \pm$ 9 milliseconds. The ejection fraction postablation improved from $46 \pm 12 \%$ to $54 \pm 10 \%$ ( $\mathrm{P}=0.01)$. Successful target sites were in the CVS alone in 3 cases, in the CVS and at the left ventricular endocardium in 5 cases, in the CVS and the aortic cusp in 1 case, the CVS and at the aortic cusp and the left ventricular endocardium in 1 case, the CVS and the endocardium and the pericardial space in 1 case, and at the left ventricular endocardium alone in 1 case. In the remaining $4 / 5$ cases, mapping was performed in the pericardial space. In 1/5 patients, the pericardial space could not be accessed. In the remaining 4 patients, pericardial mapping failed to identify a successful target site. In these patients, the CVS, the aortic cusps, the left ventricular endocardium, and the right ventricular outflow tract were mapped; in $2 / 5$ patients, radiofrequency energy was also delivered in the right ventricular outflow tract. Table 2 displays the different steps and the ablation outcome of each step. Table 2 displays the mapping and ablation data. When patients with successful ablations were compared to patients with failed ablations, the activation time at the SOO was similar in these patients ( $29 \pm 3$ vs. $34 \pm 9$ milliseconds, respectively; $\mathrm{P}=0.09$ ). However, the distance of the SOO to the second earliest anatomic structure was significantly smaller in patients with successful ablations compared to patients with unsuccessful ablations $(7.4 \pm 2.5$ vs. $14.8 \pm 2.2 \mathrm{~mm} ; \mathrm{P}=0.0001)$.

\section{SOO and Proximity to Coronary Arteries}

In $10 / 17$ patients, the SOO was estimated to be $<4 \mathrm{~mm}$ to the closest coronary arteries and radiofrequency energy could not be delivered at the SOO. In 1 patient, in whom the SOO was in the middle cardiac vein, the vein was too close to the coronary artery along its entire course; only an endocardial ablation was performed that resulted in elimination of the VA. Withdrawal of the ablation catheter to a safe distance 
TABLE 2

Characteristics of Mapping and Ablation Data of the Study Patients

\begin{tabular}{|c|c|c|c|c|c|c|c|}
\hline Patient & $\begin{array}{c}\text { PVC } \\
\text { Morphol }\end{array}$ & $\begin{array}{c}\text { LAT CVS } \\
\text { (milliseconds) }\end{array}$ & $\begin{array}{c}\text { LAT Adjacent } \\
\text { Site (milliseconds) }\end{array}$ & $\begin{array}{l}\text { Distance } \\
\quad(\mathbf{m m})\end{array}$ & $\begin{array}{c}\text { RF } \\
\text { Delivered }\end{array}$ & $\begin{array}{c}\text { Successful } \\
\text { Acute/Chronic }\end{array}$ & $\begin{array}{c}\text { RF } \\
\text { Total (min) }\end{array}$ \\
\hline 1 & RBIA & -30 & -24 & 5.9 (LV endo) & CVS+Endo+cusp & Yes/yes & 16 \\
\hline 2 & RBIA & -40 & -18 & 14.4 (LV endo) & CVS+Endo+Epic & No/no & 24 \\
\hline 3 & LBIA & -35 & -21 & 15.6 (LV endo) & CVS+Endo & No/no & 32 \\
\hline 4 & LBIA & -30 & -17 & 7.6 (LV endo) & CVS+Endo & Yes/yes & 10.5 \\
\hline 5 & RBIA & -25 & -21 & 10 (LV endo) & CVS+Endo & Yes/yes & 14 \\
\hline 6 & RBIA & -35 & -32 & 17.6 (RVOT) & CVS+Endo+Epic & $\mathrm{No} / \mathrm{no}$ & 21 \\
\hline 7 & RBIA & -22 & NA & 15 (LV endo) & CVS+Endo & $\mathrm{No} / \mathrm{no}$ & 8 \\
\hline 8 & LBIA & -30 & NA & NA & CVS & Yes/yes & 6 \\
\hline 9 & LBSA & -27 & -26 & 3.6 (LV endo) & Endo & Yes/yes & 16 \\
\hline 10 & LBSA & -27 & NA & NA & CVS & Yes/yes & 11 \\
\hline 11 & RBIA & -30 & NA & NA & CVS & Yes/yes & 7 \\
\hline 12 & RBBIA & -25 & -17 & 6.4 (LV endo) & CVS+Endo & Yes/yes & 15 \\
\hline 13 & RBIA & -21 & -19 & 7.5 (LV endo) & CVS+Endo & Yes/yes & 14 \\
\hline 14 & RBIA & -30 & -22 & 11.6 (LV endo) & CVS+Endo & Yes/yes & 10 \\
\hline 15 & RBIA & -30 & -23 & 11.5 (RVOT) & CVS+Endo & $\mathrm{No} / \mathrm{no}$ & 34 \\
\hline 16 & LBIA & -31 & -28 & 5 (cusp) & CVS+Cusp & Yes/yes & 14 \\
\hline 17 & RBIA & -26 & -21 & 9.1 (LV epi) & CVS+Epic & Yes/yes & 35 \\
\hline
\end{tabular}

CVS = coronary venous system; Epic = epicardial; LAT = local activation time; LBIA = left bundle branch block inferior axis; LBSA = left bundle branch block superior axis; $\mathrm{LV}$ endo = left ventricular endocardium; $\mathrm{LV}$ epi $=$ left ventricular epicardium; RBIA = right bundle branch block inferior axis; RF $=$ radiofrequency energy; RVOT $=$ right ventricular outflow tract.

from the coronary arteries was performed in the remaining $9 / 10$ patients, and radiofrequency energy was delivered at this location. Additional radiofrequency ablation was performed at adjacent anatomic structures and eliminated the targeted VA in 5/10 patients. In the remaining 4 patients, the ablation procedure failed.

\section{Epicardial Mapping and Ablation Within the Pericardial Space via Subxyphoid Approach}

This was attempted in $6 / 17$ patients. In $1 / 6$ patients, pericardial access could not be obtained. The earliest timing of the mapping procedure within the pericardial space was $35.5 \pm 7$ milliseconds and was no different from the earliest timing within the CVS $(-35 \pm 0.8 ; \mathrm{P}=0.9)$. This approach was effective in eliminating the targeted PVC in only $1 / 6$ patients. In this patient, the SOO was located $8.1 \mathrm{~mm}$ from the visceral pericardium and $6.6 \mathrm{~mm}$ from the left ventricular endocardium. The CVS was $4.3 \mathrm{~mm}$ from the SOO. Radiofrequency energy was delivered from the CVS, the left ventricular endocardium, and the pericardial space. Among the patients with a failed subxyphoid epicardial approach, the SOO was located $11.2 \pm 5.5 \mathrm{~mm}$ from the visceral pericardium and $8.9 \mathrm{~mm}$ from the left ventricular endocardium.

\section{SOO and Distance to Surrounding Anatomic Structures}

The closest distance of the SOO to any anatomic structure where an ablation catheter could be positioned was the CVS, which was at a mean distance of $2.1 \mathrm{~mm}$ from the SOO (the epicardial myocardium); the second closest site to the SOO was the left ventricular endocardium (mean of $7.7 \pm 2.7$ $\mathrm{mm}$ ), followed by the visceral pericardium (mean $9.7 \pm 3.7$ $\mathrm{mm}$ ). The aortic valve was furthest away from the SOO of all the measured sites (mean $15.7 \pm 5.9 \mathrm{~mm}$ ). The CVS was at a significantly shorter distance from the SOO than either left ventricular endocardium or left ventricular epicardium or aortic cusp (paired comparisons; each $\mathrm{P}<0.0001$ ).

In 2 patients, mapping was performed within the middle cardiac vein. In these 2 patients, the distance between the CVS and the epicardial myocardium at the SOO was 1 and
$3.1 \mathrm{~mm}$, whereas the distance to the visceral pericardium and the epicardial myocardium at the SOO was 6.4 and 8.7 $\mathrm{mm}$. The distance of the catheter location at the SOO to the coronary artery was 0.2 and $1.5 \mathrm{~mm}$ and an endocardial ablation was carried out effectively in 1 patient.

\section{Anatomic Relations Between Adjacent Structures Along the Course of the CVS (Tables S1 and S2)}

The mean distance of the CVS to the epicardial myocardium was $3.1 \pm 1.2 \mathrm{~mm}$ along the course of the CVS with a range of 2 to $6 \mathrm{~mm}$. The mean distance of the epicardial surface of the myocardium to the left ventricular endocardium was $8.4 \pm 2.7 \mathrm{~mm}$, with a range of $4.2-11.2 \mathrm{~mm}$. The distance between the epicardial and endocardial myocardium along the proximal GCV up to the bifurcation of the left main coronary artery was significantly shorter compared to the distal segment along the AIV $(6.4 \pm 4.3$ vs. $10.6 \pm 0.4$ $\mathrm{mm}, \mathrm{P}<0.001)$.

\section{CVS and Proximity to Coronary Arteries}

At the SOO, the CVS was at a distance of $1.5 \pm 2 \mathrm{~mm}$ from the closest coronary artery. The coronary arteries were in close proximity to the CVS (mean distance of $3.3 \pm 2.6$ $\mathrm{mm}$, range 1.1 to $10.3 \mathrm{~mm}$ ) except near the bifurcation of the left main coronary artery. At the level of the bifurcation, the mean distance was $10.3 \pm 8 \mathrm{~mm}$; at $5 \mathrm{~mm}$ distal to the bifurcation, the mean distance to the closest coronary artery was $5.9 \pm 4.2 \mathrm{~mm}$; at $5 \mathrm{~mm}$ proximal to the bifurcation, the closest coronary artery to the CVS was at a distance of 5.5 $\pm 6.3 \mathrm{~mm}$.

\section{ECG Characteristics}

All VAs with an origin within the GCV/AIV had an inferior axis and a left bundle branch block or right bundle branch block morphology depending on the origin within the GCV. ECG characteristics were different depending on whether the SOO was at the level of the bifurcation of the left main coronary artery and beyond compared to a location within the more proximal GCV. All patients with an origin within the 
middle cardiac vein had a left bundle branch block, superior axis morphology. Analyzed parameters did not correlate with procedural failure or success (Table S3).

\section{Delivery of Radiofrequency Energy}

In 3 patients, there was an impedance $>200$ Ohms and with delivery of radiofrequency energy, the temperature measured at the catheter tip immediately rose to $>50^{\circ} \mathrm{C}$, resulting in termination of radiofrequency energy delivery. In the first 2 cases, a cryocatheter was used to deliver cryoenergy at the SOO and this resulted in elimination of the targeted arrhythmia in 1 case and in the second case, radiofrequency energy from the endocardium resulted in elimination of the patients' arrhythmia. In 3 patients, radiofrequency energy was delivered at the SOO within the CVS with up to $30 \mathrm{~W}$. During ablation, however, there was no decrease of impedance and the ablation failed to eliminate the VA. In $2 / 3$ patients, the VA was eliminated by radiofrequency energy delivery from adjacent structures.

A total mean of $22 \pm 14$ minutes of radiofrequency energy was delivered. In 6/17 patients, multiple VAs were targeted; the ablation time for only the epicardial arrhythmias was 17 \pm 9 minutes. The RF time tended to be less if the arrhythmia could be ablated within the CVS compared to arrhythmias requiring ablation from multiple sites $(8 \pm 3$ vs. $18 \pm 9$ minutes; $\mathrm{P}=0.07)$. The mean procedure duration was 377 \pm 134 minutes.

\section{Follow-Up}

No procedural complications occurred. The PVC burden was reduced from $28 \pm 9 \%$ to $2.8 \pm 4.4 \%$ at 3 months $(\mathrm{P}<$ $0.0001)$.

\section{Discussion}

\section{Anatomic Correlations at the SOO}

Proximity to the epicardial SOO is key in eliminating VAs originating from this location. The CVS is embedded in the epicardial fat and is closer to the epicardial myocardium where arrhythmias arise than the visceral surface of the pericardium that covers the epicardial fat. This may explain why an epicardial ablation in the pericardial space was effective in only $1 / 6$ patients in whom it was attempted. This approach failed when the epicardial fat was $>10 \mathrm{~mm}$ from the SOO. A prior study ${ }^{5}$ also reported a lower success rate for ablation of idiopathic VAs using a subxyphoid pericardial approach. ${ }^{6}$ These results indicate that a subxyphoid pericardial approach should not be used as the initial approach for idiopathic VAs and should be reserved for cases in which other target sites including the CVS are ineffective.

Proximity to the SOO is not the only factor important for successful ablation of these arrhythmias. This study highlights other anatomic factors that play a role in the outcome of the procedure. The location of the coronary arteries along the course of the GCV limited access to the SOO of the VA in more than half of the patients and necessitated catheter withdrawal and delivery of radiofrequency energy at other sites. This resulted in an increase in the amount of radiofrequency energy that was delivered in order to reach the SOO. The proximity of the SOO to the coronary arteries rather than imprecise mapping was the most frequent cause of failed ab- lation in this study. If, however, the SOO was within $1 \mathrm{~cm}$ of an adjacent structure where radiofrequency energy could be delivered, this usually resulted in successful ablation in most instances. If this was not the case, ablation was often unsuccessful.

A pace-map with $\geq 10 / 12$ matching leads was used as evidence that the ablation catheter was in contact with the SOO. Ideally, a perfect pace-match should be present at the SOO; however, most often high output is required for ventricular capture within the CVS, and this can result in a larger amount of tissue that is captured. Therefore, we used a pace-map score of $\geq 10 / 12$ matching leads rather than $12 / 12$ matching pace-maps.

Measurements along the GCV indicate that the longest distance to the coronary arteries from within the GCV is within $1 \mathrm{~cm}$ of the bifurcation of the left main coronary artery.

The proximity to the aortic valve is closest at $1 \mathrm{~cm}$ proximal to the left main bifurcation, suggesting that in some patients in whom an epicardial arrhythmia originates from this area, an approach through the left aortic cusp may help to target such a focus.

The distance of the epicardial myocardium to the endocardium was shorter along the basal-lateral left ventricle as compared to the anterior free wall, and it was a mean of 6.4 $\mathrm{mm}$ up to the bifurcation of the left main coronary artery. Therefore, epicardial arrhythmias that arise from the basallateral left ventricular epicardium also can be reached from within the left ventricular endocardium; the more lateral, the smaller the distance between the ventricular epicardium and endocardium.

Because of the close proximity of the SOO to a coronary artery in 4 patients, the short distance of the SOO to the left ventricular endocardium allowed for safe applications of radiofrequency energy at these sites to reach the SOO. This was only possible because of the close anatomic relationship that the SOO had with these structures.

The tapering diameter of the CVS may be responsible for the inability to deliver sufficient radiofrequency energy to the SOO in the distal part of the GCV or the AIV. This may result in a high impedance or a rapid rise in temperature during radiofrequency energy delivery despite the use of an irrigated-tip catheter. The diameter of the open-tip irrigated catheter is $2.5-2.7 \mathrm{~mm}$, which approximates the diameter of the distal CVS. A lack of surrounding blood flow renders delivery of radiofrequency energy difficult even when open irrigation is used. Cryoablation or positioning the catheter in an anatomic structure adjacent to the SOO can help to address this problem.

In order to better use the proximity of the CVS to the epicardial myocardium for effective ablation, current catheter technology needs to be improved. Specifically, catheter flexibility needs to be improved to reach more distal sites. A preprocedural CT angiogram may be beneficial in order to better plan an ablation procedure in patients with epicardial VAs.

The ECG was helpful to indicate the relative location of the SOO in the GCV but failed to indicate patients with unsuccessful procedures.

\section{Middle Cardiac Vein and Surrounding Structures}

The data obtained for the middle cardiac vein essentially reflect the measurements along the GCV and the AIV. 
Especially, the distance from the visceral pericardium to the underlying epicardial myocardium is in the $1.5 \mathrm{~cm}$ range in the proximal part of the middle cardiac vein, whereas the distance form the CVS to the myocardium is in the 2-4 mm range. On the other hand, the proximity to the coronary arteries can be within 1-3 mm along most of the course of the middle cardiac vein.

\section{Prior Publications}

Yokokawa et al. as well as Abularch et al. demonstrated the need for endocardial ablations or ablations form the aortic cusps to target idiopathic, epicardial VAs. ${ }^{3,7}$ This study extends the findings of these prior reports by correlating anatomic structures and landmarks with electrophysiologic findings during ablation procedures of these VAs.

\section{Limitations}

The procedure was unsuccessful in 5/17 patients and it is possible that the SOO was not correctly identified in these patients. However, only patients with early activation times in whom matching pace-maps within the CVS or the epicardium could be identified were included in this analysis. Intramural origins were less likely since matching pace-maps were obtained from the CVS.

\section{Clinical Implications}

Idiopathic VAs originating from the left ventricular epicardium should be approached predominantly from the CVS or adjacent structures including the left ventricular endocardium or the aortic cusps. A subxyphoid pericardial approach most often fails due to epicardial fat separating the ablation catheter from the epicardial myocardium. A CT angiogram of the coronary arteries can help to assess the anatomical relationship of the CVS to adjacent structures, thereby facilitating the ablation procedure.

\section{References}

1. Schweikert RA, Saliba WI, Tomassoni G, Marrouche NF, Cole CR, Dresing TJ, Tchou PJ, Bash D, Beheiry S, Lam C, Kanagaratnam L, Natale A: Percutaneous pericardial instrumentation for endo-epicardial mapping of previously failed ablations. Circulation 2003;108:13291335.

2. Baman TS, Ilg KJ, Gupta SK, Good E, Chugh A, Jongnarangsin K, Pelosi F Jr, Ebinger M, Crawford T, Oral H, Morady F, Bogun F: Mapping and ablation of epicardial idiopathic ventricular arrhythmias from within the coronary venous system. Circ Arrhythm Electrophysiol 2010;3:274-279.

3. Yokokawa M, Latchamsetty R, Good E, Chugh A, Pelosi F Jr, Crawford T, Jongnarangsin K, Oral H, Morady F, Bogun F: Ablation of epicardial ventricular arrhythmias from nonepicardial sites. Heart Rhythm 2011;8:1525-1529.

4. D'Avila A, Gutierrez P, Scanavacca M, Reddy V, Lustgarten DL, Sosa E, Ramires JA: Effects of radiofrequency pulses delivered in the vicinity of the coronary arteries: Implications for nonsurgical transthoracic epicardial catheter ablation to treat ventricular tachycardia. Pacing Clin Electrophysiol 2002;25:1488-1495.

5. Desjardins B, Morady F, Bogun F: Effect of epicardial fat on electroanatomical mapping and epicardial catheter ablation. J Am Coll Cardiol 2010;56:1320-1327.

6. Tung R, Michowitz Y, Yu R, Mathuria N, Vaseghi M, Buch E, Bradfield J, Fujimura O, Gima J, Discepolo W, Mandapati R, Shivkumar K: Epicardial ablation of ventricular tachycardia: An institutional experience of safety and efficacy. Heart Rhythm 2013;10:490-498.

7. Jauregui Abularach ME, Campos B, Park KM, Tschabrunn CM, Frankel DS, Park RE, Gerstenfeld EP, Mountantonakis S, Garcia FC, Dixit S, Tzou WS, Hutchinson MD, Lin D, Riley MP, Cooper JM, Bala R, Callans DJ, Marchlinski FE: Ablation of ventricular arrhythmias arising near the anterior epicardial veins from the left sinus of valsalva region: ECG features, anatomic distance, and outcome. Heart Rhythm 2012;9:865-873.

\section{Supporting Information}

Additional supporting information may be found in the online version of this article at the publisher's website:

Table S1: Serial Measurements of Adjacent Anatomic Structures Along the Great Cardiac Vein.

Table S2: Serial Measurements of Adjacent Anatomic Structures Along the Middle Cardiac Vein.

Fig S1: Volume rendered 3-D reconstruction of the CTA with the registered catheter electrodes within the great cardiac vein close to the left main bifurcation (LM bifurcation).

Fig S2: Schema indicating the step-wise approach performed in mapping and ablation of the patients ventricular arrhythmias as well as the results of each step. CVS $=$ coronary venous system; $\mathrm{VA}=$ ventricular arrhythmias; $\mathrm{RF}=$ radiofrequency energy; $\mathrm{LV}=$ left ventricular. 